\title{
Successful twin pregnancy in a 38-year-old woman with Pompe disease despite interruption of enzyme replacement therapy (ERT)
}

\author{
N Tiling*, L Bosanska, G Gossing, H Krieg, A Krebs, S Rosseau, H Schütte, K Irlbacher, U Plöckinger \\ From Proceedings of the 6th European Symposium: Steps Forward in Pompe Disease \\ Berlin, Germany. 23-24 November 2012
}

\section{Introduction}

Clinical features of Pompe disease are limb-girdle muscle weakness and respiratory insufficiency. Data on pregnancy with or without ERT are rare. Herein, we report on a twin pregnancy in a 38-year-old primiparous who declined ERT during pregnancy.

\section{Results}

After successful insemination of 2 foetuses, ERT was stopped at the patient's request. Investigations were performed before and during pregnancy until week 30 . The Walton/Gardner Medwin Scale was stable during the preceding $2 \mathrm{y}$ on ERT and during pregnancy. Echocardiography demonstrated normal left-ventricular function. No changes were observed in creatine kinase (CK) levels, GAA activity, or quality of life (SF-36). In contrast, pulmonary and functional scores clearly deteriorated: FEV1 $(58 \% \rightarrow 54 \%)$ and supine VC (61 $\rightarrow 55 \%) ; 6$-min walk test $324 \rightarrow 240 \mathrm{~m}$ (73\%); climbing 4 steps $4 \rightarrow 5.1 \mathrm{sec}(128 \%)$; standing from supine $4.5 \rightarrow 19 \mathrm{sec}$ ( $433 \%$; due to back pain); 10m walking $8 \rightarrow 10 \mathrm{~m}$ (125\%). Growth-curves for the dichorionicdiamniote twins were normal. At week 34, HELLP-syndrome (hemolysis, elevated liver function test, low platelet counts) was diagnosed due to increased ALT $618 \mathrm{U} / \mathrm{l}(\mathrm{N}<35)$, reduced thrombocyte count $[95 / \mathrm{nl}$ $(\mathrm{N}>150)]$ and peripheral edema. Subsequently, a cesarean section was performed under combined spinal epidural anesthesia. No intra or postoperative pulmonary complications occurred. Two mature, healthy boys, with normal GAA were delivered. Peripartal evaluation of the patient's GAA showed a uniquely normal level.

\section{Discussion}

We describe the first in vitro fertilisation and twin pregnancy in a patient with Pompe disease with interruption of ERT during pregnancy. The observed clinical deterioration may have resulted from a combination of treatment interruption and pregnancy-related restrictions on pulmonary/motor function. The patient's back pain may be related to an exacerbated strain on weak spinal muscles due to the twin pregnancy. No transfer of GAA from the foetus to the mother was observed during pregnancy, but peripartal transfer occurred. HELLP-Syndrome is thought to be unrelated to Pompe since it is a common complication in elderly primiparous.

\section{Conclusion}

For a patient that has a stable and reasonably good status before pregnancy, the interruption of ERT may be possible without deleterious effects. However, close follow-up is required.

Published: 29 May 2013

\section{doi:10.1186/1471-2474-14-S2-P8}

Cite this article as: Tiling et al:: Successful twin pregnancy in a 38-yearold woman with Pompe disease despite interruption of enzyme replacement therapy (ERT). BMC Musculoskeletal Disorders 2013 14(Suppl 2):P8.

Charité Universitätsmedizin Berlin, Berlin, Germany

(c) 2013 Tiling et al; licensee BioMed Central Ltd. This is an Open Access article distributed under the terms of the Creative Commons 